\title{
Localized Proton NMR Spectroscopy in Different Regions of the Human Brain in Vivo. Relaxation Times and Concentrations of Cerebral Metabolites
}

\author{
J. Frahm, H. BRUHN, M. L. GYNGELL, K. D. MERBOLDT, \\ W. HÄNICKE, AND R. SAUTER* \\ Max-Planck-Institut für biophysikalische Chemie, Postfach 2841, D-3400 Göttingen, Federal Republic \\ of Germany; and ${ }^{*}$ Bereich Medizinische Technik, Siemens AG, Henkestrasse 127, \\ D-8520 Erlangen, Federal Republic of Germany
}

Received August 1, 1988; revised October 25, 1988

\begin{abstract}
High-resolution proton NMR spectra of normal human brain in vivo have been obtained from selected 27 - and 64-ml volumes-of-interest (VOI) localized in the insular area, the occipital area, the thalamus, and the cerebellum of normal volunteers. Localization was achieved by stimulated echo (STEAM) sequences using a conventional 1.5-T whole-body MRI system (Siemens Magnetom). The proton NMR spectra show resonances from lipids, lactate, acetate, $N$-acetylaspartate (NAA), $\gamma$-aminobutyrate, glutamine, glutamate, aspartate, creatine and phosphocreatine, choline-containing compounds, taurine, and inositols. While $T_{1}$ relaxation times of most of these metabolites were about $1100-1700 \mathrm{~ms}$ without significant regional differences, their $T_{2}$ relaxation times varied between 100 and $500 \mathrm{~ms}$. The longest $T_{2}$ values of about $(500 \pm 50) \mathrm{ms}$ were observed for the methyl protons of NAA in the white matter of the occipital lobe compared to $(320 \pm 30) \mathrm{ms}$ in the other parts of the brain. No significant regional $T_{2}$ differences were found for choline and creatine methyl resonances. The relative concentrations of NAA in gray and white matter were found to be $35 \%$ higher than those in the thalamus and cerebellum. Assuming a concentration of $10 \mathrm{~m} M$ for total creatine the resulting NAA concentrations of 13-18 $\mathrm{mM}$ are by a factor of 2-3 higher than previously reported using analytical techniques. Cerebral lactate reached a maximum concentration of about 1.0 mM. 1989 Academic Press, Inc.
\end{abstract}

\section{INTRODUCTION}

Complementary to anatomical and functional studies by magnetic resonance imaging (MRI), localized in vivo NMR spectroscopy opens the way to a biochemical characterization of tissues and their diseased states. Noninvasive insights may be gained into the metabolism of a variety of compounds within the whole living organism. Moreover, many biochemical and physiological questions may now be investigated in human subjects rather than in animals, avoiding the need of anesthesia and associated physiological complications as well as the uncertainties in establishing a correlation between animal studies and human applications.

In particular, proton NMR spectroscopy is expected to broaden our understanding of metabolic processes in vivo. The abundant representation of protons in molecules 
FRAHM ET AL.
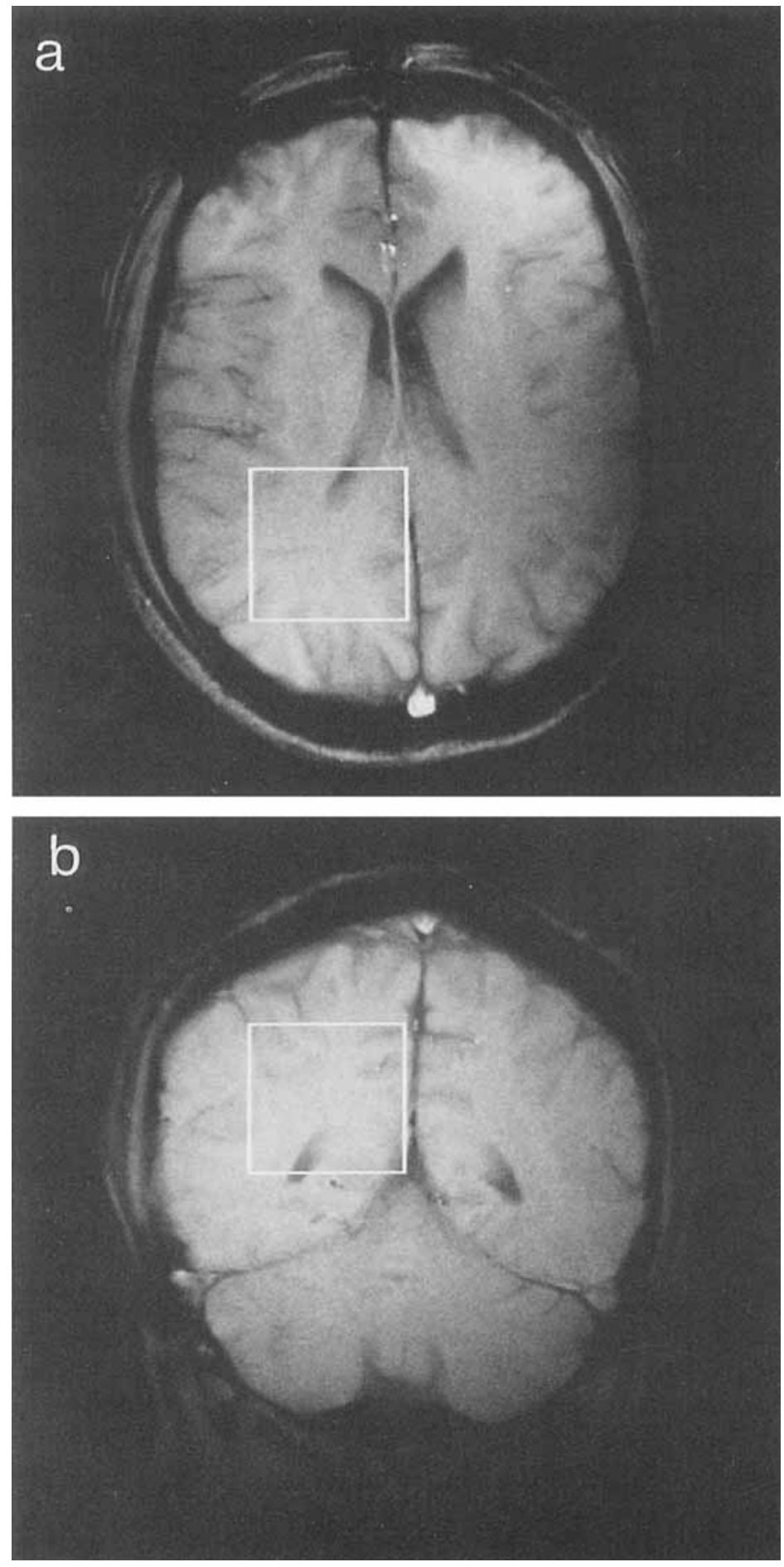


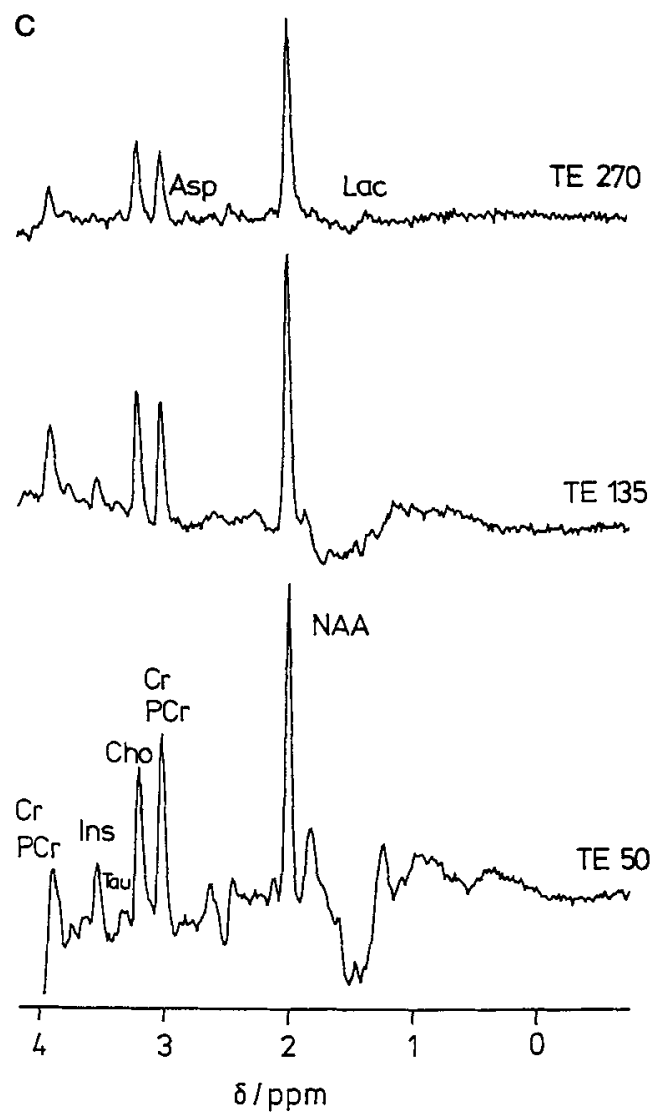

FIG. 1. Sixty-four-megahertz (1.5-T) FLASH MR images $\left(40^{\circ}, \mathrm{TR}=100 \mathrm{~ms}\right.$, TE $\left.=11 \mathrm{~ms}\right)$ and localized proton NMR spectra of a normal volunteer from a $4 \times 4 \times 4 \mathrm{~cm}^{3}$ VOI in the occipital area of the brain selected for in vivo spectroscopy of predominantly white matter. (a) Axial image, (b) coronal image, and (c) series of spectra at echo times $\mathrm{TE}=50,135$, and $270 \mathrm{~ms}$, respectively, demonstrating the $T_{2}$ dependence of the individual metabolites ( $64 \mathrm{ml}, \mathrm{TR}=1500 \mathrm{~ms}, 512$ scans). Assignments of metabolites include lactate (Lac), $N$-acetylaspartate (NAA), aspartate (Asp), creatine $(\mathrm{Cr})$ and phosphocreatine (PCr), cholinecontaining compounds (Cho), taurine (Tau), and inositols (Ins).

of biological interest gives access to the pool of free amino acids, neurotransmitters, fatty acids, and carbohydrates as well as to compounds related to the energy metabolism (see, e.g., Ref. (I)). In addition, the high sensitivity of proton NMR lends itself to much shorter measuring times and smaller measuring volumes than required for equivalently localized phosphorous studies. Thus, more extensive spectroscopic protocols that allow the determination of relaxation effects needed for any attempt to quantify spectroscopic findings may be recorded.

An important aspect of in vivo NMR studies is a reliable spatial localization. In preceding papers (2-4) we have demonstrated that stimulated echo (STEAM) spectroscopy provides a simple solution that is particularly suited to proton NMR. This 
has been confirmed by preliminary applications of the technique to patients with brain tumors $(5,6)$ and cerebral infarctions $(7)$. No spectral contamination with resonances from normal brain tissue has been observed when the volume-of-interest (VOI) was fully localized inside the lesion. In this paper we report a more detailed study of cerebral metabolites that are currently accessible from localized proton NMR spectra of healthy volunteers. $T_{1}$ and $T_{2}$ relaxation times as well as relative and absolute in vivo concentrations of metabolite resonances are determined in various regions of the brain and compared to the results of in vitro studies obtained using analytical techniques.

\section{METHODS}

The STEAM spectroscopy technique allows a reliable selection of a VOI as well as suppression of the unwanted water signal in a single experimental step. Its implementation on a conventional whole-body MRI system (Siemens Magnetom) used here has been described in detail in a preceding paper (4). The sequence comprises three slice selective RF pulses applied in the presence of magnetic field gradients according to

$$
90^{\circ}(\mathrm{CHESS})-90^{\circ}(\# 1)-\mathrm{TE} / 2-90^{\circ}(\# 2)-\mathrm{TM}-90^{\circ}(\# 3)-\mathrm{TE} / 2-\mathrm{STE} .
$$

Localization is accomplished by the fact that the acquired stimulated echo (STE) NMR signal originates only from spins that experience all three pulses. These spins are defined by the intersection of the three corresponding slices. Water suppression is achieved by preceding chemical-shift-selective (CHESS) pulses. Exciting only a narrow frequency range $( \pm 0.4 \mathrm{ppm})$ around the water resonance $(4.7 \mathrm{ppm})$, the resulting transverse magnetizations are spoiled. Thus, the slice-selective RF pulses of the STEAM sequence uniformly excite all other spectral ranges. This is an important advantage for quantitative evaluations. In the present study the middle interval TM was fixed to $30 \mathrm{~ms}$, whereas the repetition time TR and the echo time TE were varied to obtain different $T_{1}$ and $T_{2}$ weightings, respectively. A total of 14 healthy volunteers (male, 20-35 years) were investigated in five studies of the occipital area and the occipital lobe, and three studies of the insular area, the thalamus, and the cerebellum. Image selection as well as control of proper positions and dimensions of the VOI, either $3 \times 3 \times 3$ or $4 \times 4 \times 4 \mathrm{~cm}^{3}$, was performed in all three orientations using an interleaved three-slice FLASH MRI sequence.

\section{RESULTS}

Figure 1 shows FLASH MR images and localized proton NMR spectra of the brain of a normal volunteer. The $4 \times 4-\mathrm{cm}^{2}$ squares in the axial and coronal images (Figs. $1 \mathrm{a}$ and $\mathrm{lb}$ ) define a 64-ml VOI in the occipital area which has been selected for spectroscopy of predominantly white matter. The series of water-suppressed proton spectra (Fig. 1c) demonstrates the $T_{2}$ dependence of individual metabolite resonances using TE values of 50,135 , and $270 \mathrm{~ms}$ ( $\mathrm{TR}=1500 \mathrm{~ms}, 512$ scans). Correspondingly, 


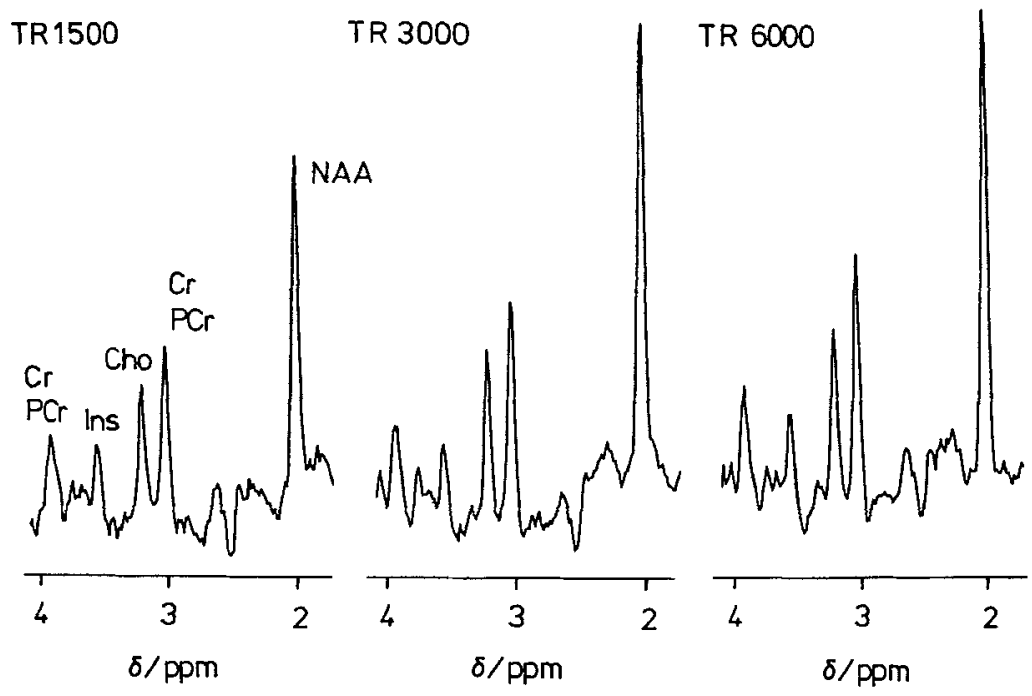

FIG. 2. Sixty-four-megahertz (1.5-T) localized proton NMR spectra of predominantly white matter in the occipital area of the brain of a normal volunteer as indicated in Fig. 1 . The series of spectra at repetition times $\mathrm{TR}=1500,3000$, and $6000 \mathrm{~ms}$, respectively, demonstrates the $T_{1}$ dependence of the individual metabolites ( $64 \mathrm{ml}, \mathrm{TE}=50 \mathrm{~ms}, 128 \mathrm{scans})$.

the series of spectra shown in Fig. 2 describes the $T_{1}$ dependence of metabolites in the same VOI using TR values of 1500,3000 , and $6000 \mathrm{~ms}$ (TE $=50 \mathrm{~ms}, 128$ scans). Resonance assignments $(1,4)$ are made to lactate $(\mathrm{Lac}, 1.3 \mathrm{ppm})$, acetate $(\mathrm{Ac}, 1.85$ $\mathrm{ppm}$ ), $N$-acetylaspartate (NAA, $2.0 \mathrm{ppm}$ ), glutamate (Glu, $2.1 \mathrm{ppm}$ ), $\gamma$-aminobutyrate (GABA, $2.25 \mathrm{ppm}$ ), glutamine (Gln, $2.1 \mathrm{ppm}, 2.45 \mathrm{ppm}$ ), aspartate (Asp, 2.8 $\mathrm{ppm})$, creatine and phosphocreatine $(\mathrm{Cr} / \mathrm{PCr}, 3.0 \mathrm{ppm}, 3.9 \mathrm{ppm})$, choline-containing compounds (Cho, $3.2 \mathrm{ppm}$ ), taurine (Tau, $3.3 \mathrm{ppm}$ ), and inositols (Ins, $3.5 \mathrm{ppm}$ ). Most spectral assignments (and relaxation times) have been confirmed by studies of model solutions using both the 1.5-T whole-body system with spatial localization and a conventional 7.0-T high-resolution NMR spectrometer. Further resonances with considerably lower intensities occur at 7.0-7.5 and 8.0-8.5 ppm and probably represent exchangeable protons of cytosolic proteins and aromatic protons of adenine nucleotides, respectively. Residual lipid signals $(0.5-1.5 \mathrm{ppm})$ may be due to contributions from small regions containing fat as parts of the VOI extend beyond the right hemisphere (see Fig. 1b).

Similar images and spectra have been recorded from the insular area containing predominantly gray matter (Fig. 3), the cerebellum (Fig. 4), and the thalamus (Fig. 5). For studies of the insular area and the thalamus the VOI was reduced to $27 \mathrm{ml} \mathrm{(3}$ $\left.\times 3 \times 3 \mathrm{~cm}^{3}\right)$ to ensure better localization and to reduce partial volume effects. In the thalamus it turned out to be more difficult when compared to other areas to achieve a good field homogeneity by localized shimming on the VOI. The spectral quality shown in Fig. 5 is slightly degraded due to a $6-\mathrm{Hz}$ linewidth compared to the usual 
FRAHM ET AL.

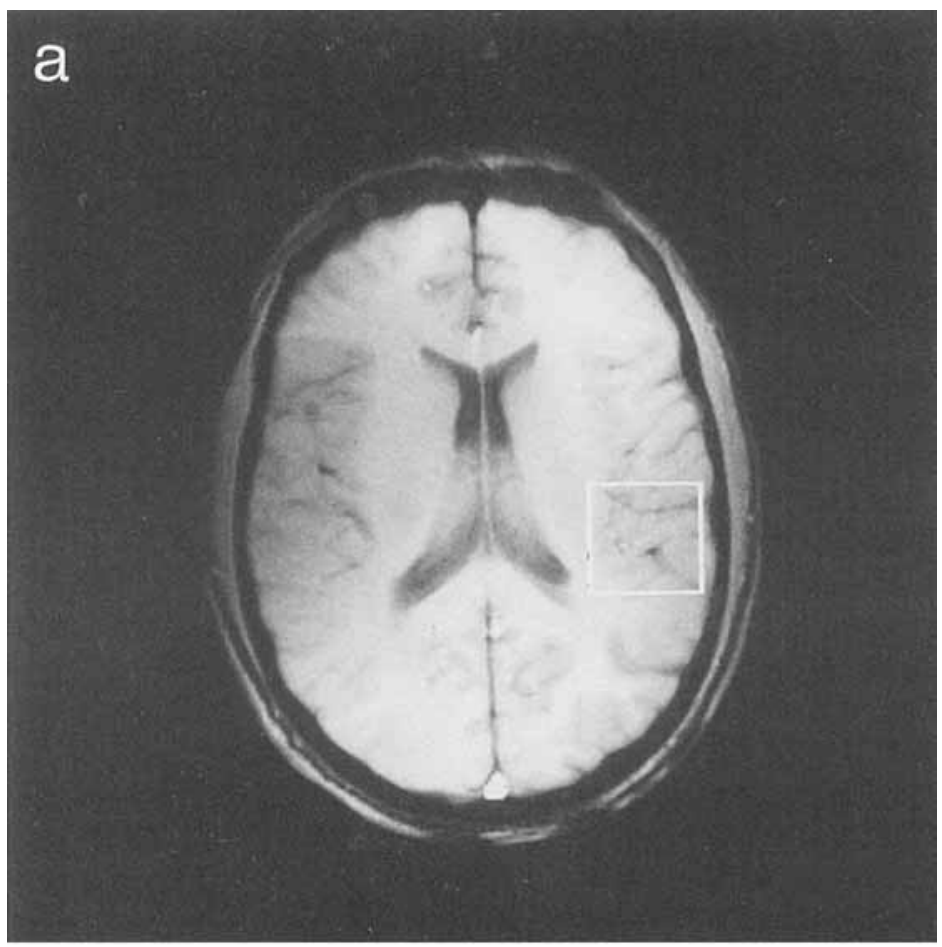

b 


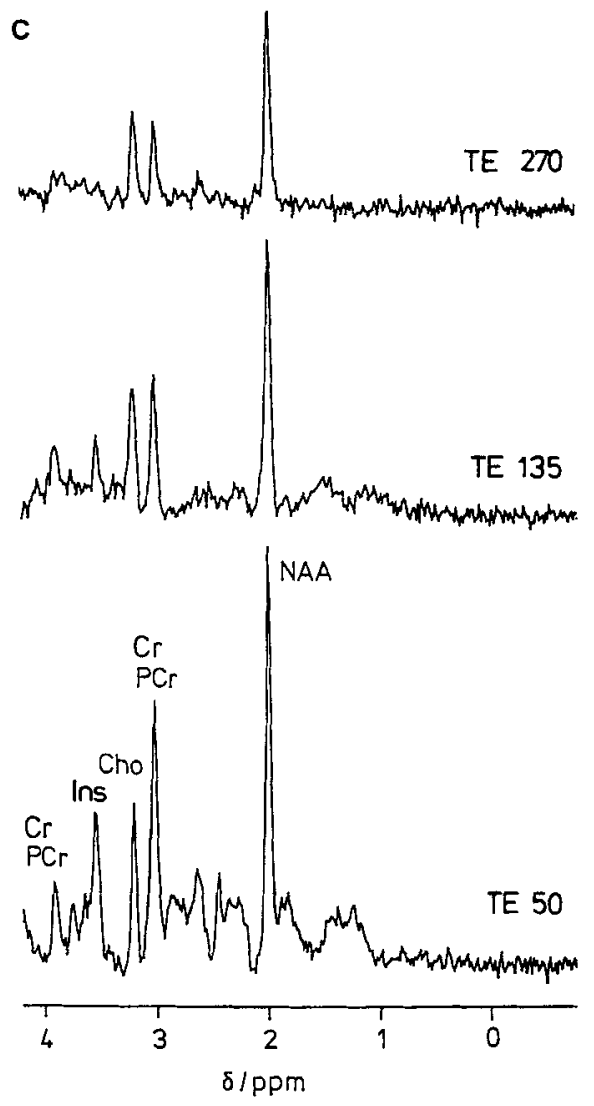

FIG. 3. Sixty-four-megahertz (1.5-T) FLASH MR images and localized proton NMR spectra of a normal volunteer from a $3 \times 3 \times 3 \mathrm{~cm}^{3} \mathrm{VOI}$ in the insular area of the brain selected for in vivo spectroscopy of predominantly gray matter. (a) Axial image, (b) coronal image, and (c) series of spectra at echo times TE $=50,135$, and $270 \mathrm{~ms}$, respectively, demonstrating the $T_{2}$ dependence of the individual metabolites ( 27 $\mathrm{ml}$ VOI, $\mathrm{TR}=1500 \mathrm{~ms}, 512$ scans).

value of about $4 \mathrm{~Hz}$. However, it is our impression that in vivo linewidths of $4-6 \mathrm{~Hz}$ are still determined by magnet inhomogeneities, shim current instabilities, and eddy current effects rather than due to natural $T_{2}$ relaxation times or biological susceptibility differences. $T_{2}$ relaxation times of the order of $0.5 \mathrm{~s}$ translate into natural linewidths of less than $1 \mathrm{~Hz}$. It should be noted that in this study no spectral processing was applied except a mild exponential multiplication of the data in the time domain.

$T_{1}$ and $T_{2}$ relaxation times of resonances from major metabolites in the investigated parts of the brain are summarized in Tables 1 and 2. The relaxation data were determined from both signal areas and peak intensities using a nonlinear GaussNewton fitting procedure for $T_{1}$ values and a linear least-squares fit for $T_{2}$ values, respectively. Although $T_{1}$ and $T_{2}$ calculations were based on only three repetition times or echo times and three to five independent volunteer studies, deviations of 
FRAHM ET AL.
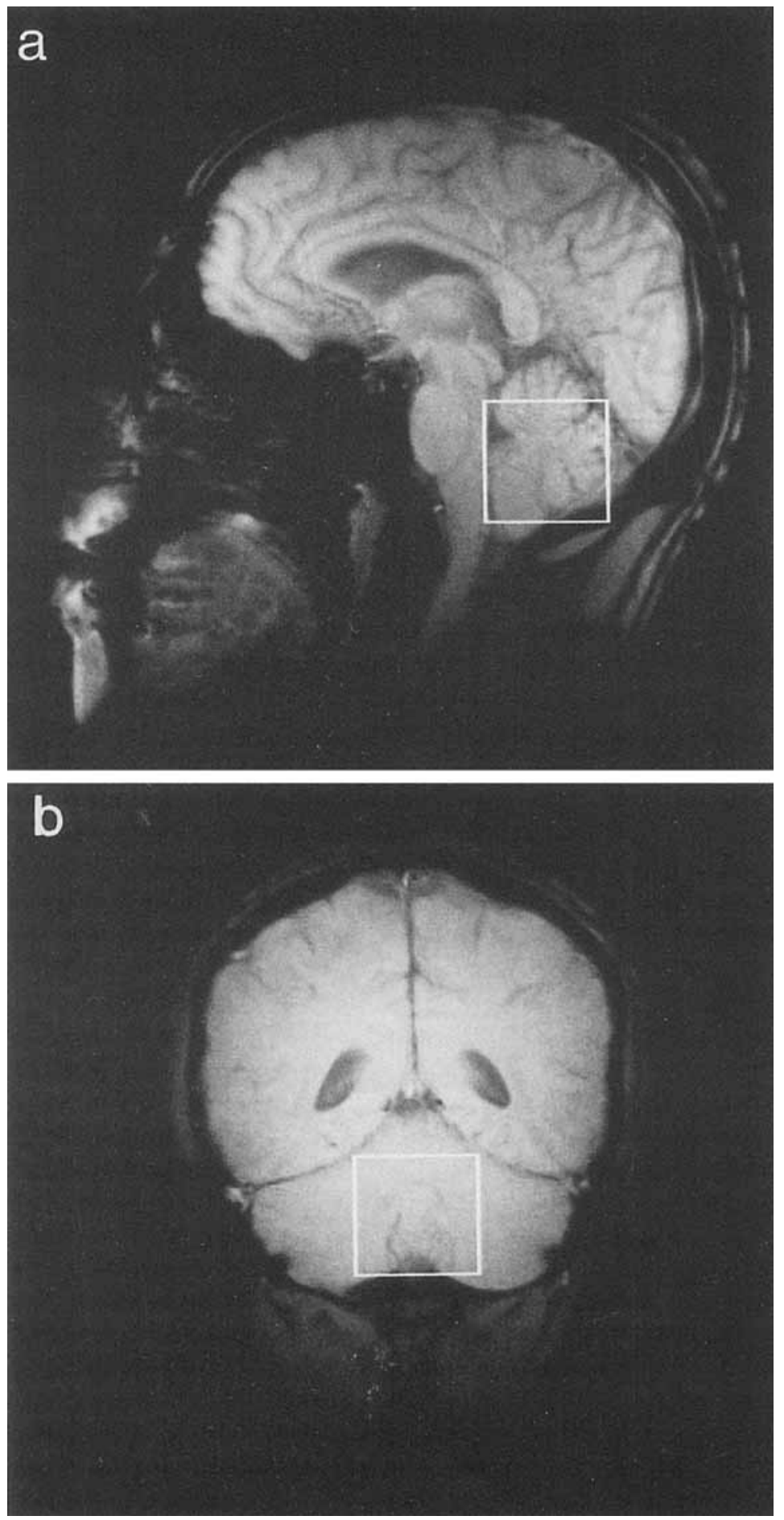

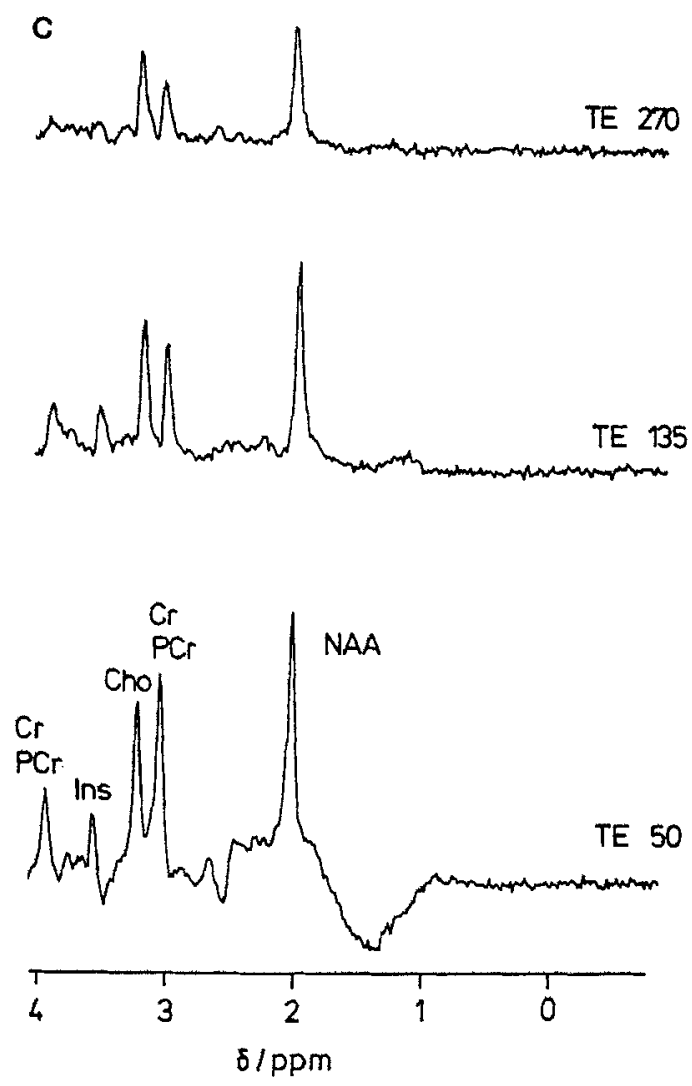

FIG. 4. Sixty-four-megahertz (1.5-T) FLASH MR images and localized proton NMR spectra of a normal volunteer from a $4 \times 4 \times 4 \mathrm{~cm}^{3}$ VOI selected for in vivo spectroscopy of the cerebellum. (a) Sagittal image, (b) coronal image, and (c) series of spectra at echo times $T E=50,135$, and $270 \mathrm{~ms}$, respectively, demonstrating the $T_{2}$ dependence of the individual metabolites $(64 \mathrm{ml} \mathrm{VOI}, \mathrm{TR}=1500 \mathrm{~ms}, 512$ scans).

individual experiments from the median values given in Tables 1 and 2 were always within a range of $\pm 10 \%$. Particularly low variations were obtained for the creatine methyl resonance. Lactate relaxation times were obtained from patients with astrocytomas and cerebral infarctions exhibiting considerably enhanced concentrations of lactic acid (5-7). Further relaxation times of metabolites with lower concentration and/or lower proton NMR signal strength such as aspartate, glutamine, glutamate, and GABA may be estimated from a qualitative comparison of spectra obtained at repetition times of 1500 and $6000 \mathrm{~ms}$ as well as at echo times of 50 and $270 \mathrm{~ms}$, respectively.

\section{DISCUSSION}

Although it is not yet clear whether or not or to which extent the in vivo relaxation times and concentrations of cerebral metabolites are influenced by the brain function 
FRAHM ET AL.

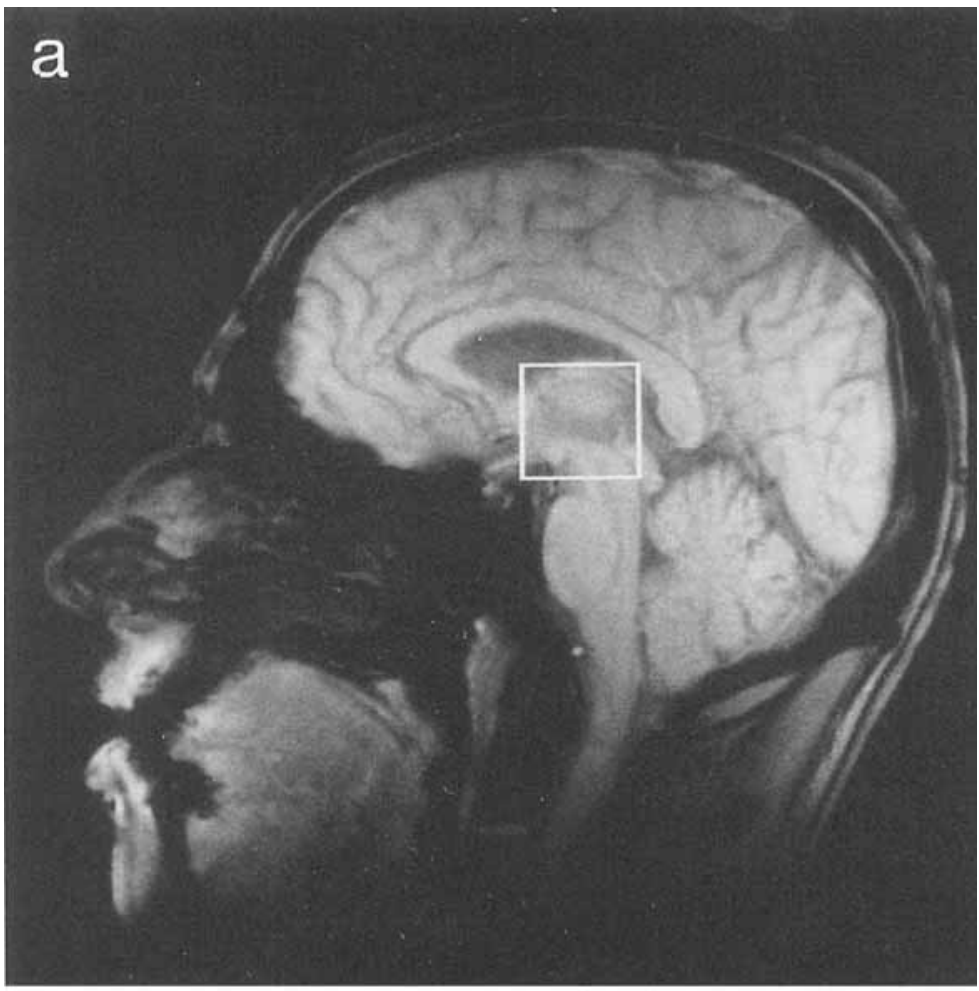

b

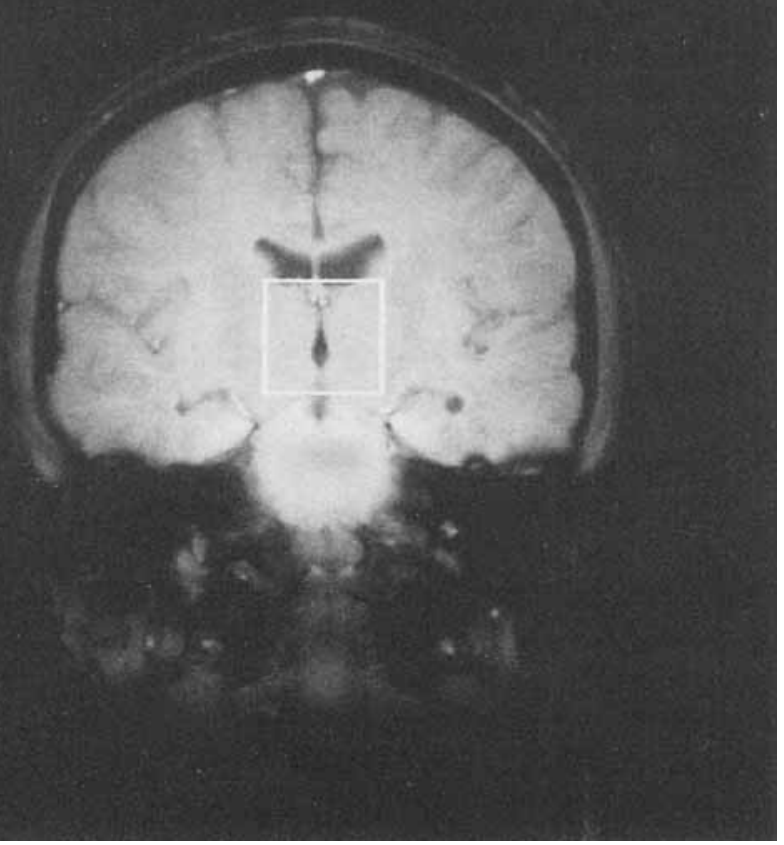



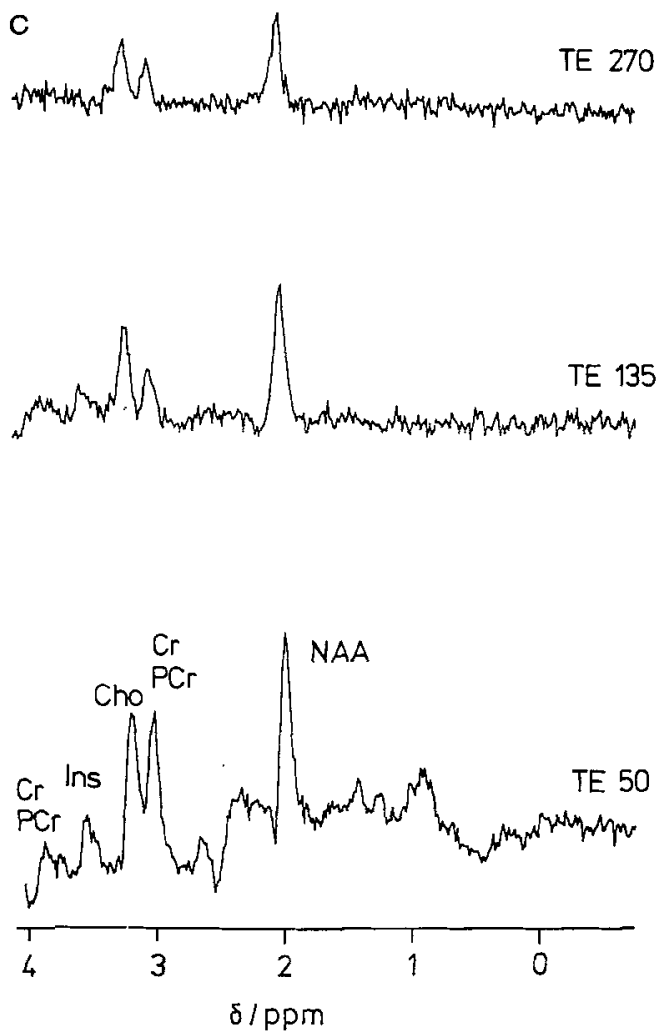

FIG. 5. Sixty-four-megahertz (1.5-T) FLASH MR images and localized proton NMR spectra of a normal volunteer from a $3 \times 3 \times 3 \mathrm{~cm}^{3}$ VOI selected for in vivo spectroscopy of the thalamus. (a) Sagittal image, (b) coronal image, and (c) series of spectra at echo times $\mathrm{TE}=50,135$, and $270 \mathrm{~ms}$, respectively, demonstrating the $T_{2}$ dependence of the individual metabolites $(27 \mathrm{ml} \mathrm{VOI}, \mathrm{TR}=1500 \mathrm{~ms}, 512$ scans $)$.

only very small differences were observed between individuals of the rather homogeneous group of volunteers studied here. In fact, the proton brain spectra turned out to be highly reproducible. The age dependence of the appearance of metabolites in proton brain spectra is the subject of a further investigation. On the other hand, a comparison of spectra recorded at the same echo time (Figs. 1c, 3c, 4c, 5c) at first glance reveals regional differences in the normal human brain. However, since these effects may be due to either variations of metabolite concentrations or relaxation times or both, an interpretation of the spectra alone may be completely misleading. In particular, a change in the ratio of signal intensities gives only a rough indication of some change in either of the parameters, while no change may cover a compensatory behavior of relaxation times and concentration. A proper elucidation and spectral interpretation requires the knowledge of $T_{1}$ and $T_{2}$ relaxation times of individual metabolite resonances.

Although $T_{1}$ and $T_{2}$ values may be obtained in solution, it remains important to measure relaxation times in vivo in view of the large number of parameters involved 
TABLE 1

Spin-Lattice Relaxation Times $T_{1}$ of Major Metabolites Detected in Localized Proton NMR Spectra of the Normal Human Brain in Vivo

\begin{tabular}{llccccc}
\hline Compound & \multicolumn{1}{c}{$\begin{array}{c}\text { Molecular } \\
\text { group }\end{array}$} & $\begin{array}{c}\delta \\
(\mathrm{ppm})\end{array}$ & $\begin{array}{c}\text { Insular } \\
\text { area }\end{array}$ & $\begin{array}{c}\text { Occipital } \\
\text { area }\end{array}$ & Thalamus & Cerebellum \\
\hline $\mathrm{Lac}$ & $\mathrm{CH}_{3}$ & 1.3 & - & 1550 & - & - \\
$\mathrm{NAA}$ & $\mathrm{CH}_{3}$ & 2.0 & 1650 & 1450 & 1400 & 1700 \\
$\mathrm{Cr}+\mathrm{PCr}$ & $\mathrm{N}-\mathrm{CH}_{3}$ & 3.0 & 1750 & 1550 & 1750 & 1500 \\
& $\mathrm{~N}-\mathrm{CH}_{2}$ & 3.9 & 1750 & 1050 & - & 1200 \\
Cho & $\mathrm{N}-\left(\mathrm{CH}_{3}\right)_{3}$ & 3.2 & 1100 & 1150 & - & 1500 \\
Tau & $\mathrm{N}-\mathrm{CH}_{2}, \mathrm{~S}-\mathrm{CH}_{2}$ & 3.3 & - & 1700 & 1100 & 1850 \\
Ins & $\mathrm{CH}(3-6)$ & 3.5 & 1350 & 900 & - \\
\hline
\end{tabular}

Note. Relaxation times are given in milliseconds. The data refer to median values with deviations of about $\pm 10 \%$.

( $\mathrm{pH}$, temperature, ions, proteins, cellular structures, etc.) and as a reference for alterations expected under pathological conditions. Possible complications may also arise from compartmentation and tissue heterogeneity. In fact, gradient-localized spectra may be further affected by $T_{2}$ relaxation times that are modulated by exchange contributions, molecular diffusion, transport processes, or even macroscopic motion and flow (3). Quantitatively reliable evaluations are mainly expected for singlet resonance signals avoiding complications from differential $J$ modulation effects on the STE signal at different echo times. In fact, a TE value of $270 \mathrm{~ms}$ was selected to provide inphase conditions for multiplets with spin-spin coupling constants $J=7 \mathrm{~Hz}$ such as observed for the lactate methyl group.

TABLE 2

Spin-Spin Relaxation Times $T_{2}$ of Major Metabolites Detected in Localized Proton NMR Spectra of the Normal Human Brain in Vivo

\begin{tabular}{llccccc}
\hline Compound & \multicolumn{1}{c}{$\begin{array}{c}\text { Molecular } \\
\text { group }\end{array}$} & $\begin{array}{c}\delta \\
(\mathbf{p p m})\end{array}$ & $\begin{array}{c}\text { Insular } \\
\text { area }\end{array}$ & $\begin{array}{c}\text { Occipital } \\
\text { area }\end{array}$ & Thalamus & Cerebellum \\
\hline Lac & $\mathrm{CH}_{3}$ & 1.3 & - & 1200 & - & - \\
NAA & $\mathrm{CH}_{3}$ & 2.0 & 330 & 450 & 340 & 300 \\
$\mathrm{Cr}+\mathrm{PCr}$ & $\mathrm{N}-\mathrm{CH}_{3}$ & 3.0 & 250 & 240 & 200 & 190 \\
& $\mathrm{~N}-\mathrm{CH}_{2}$ & 3.9 & 160 & 190 & - & 120 \\
Cho & $\mathrm{N}-\left(\mathrm{CH}_{3}\right)_{3}$ & 3.2 & 380 & 330 & 320 & 410 \\
Tau & $\mathrm{N}-\mathrm{CH}_{2}, \mathrm{~S}-\mathrm{CH}_{2}$ & 3.3 & - & 270 & - & 130 \\
Ins & $\mathrm{CH}(3-6)$ & 3.5 & 130 & 110 & 150 \\
\hline
\end{tabular}

Note. Relaxation times are given in milliseconds. The data refer to median values with deviations of about $\pm 10 \%$. 


\section{$T_{1}$ Relaxation Times}

NMR relaxation times reflect mobility at the molecular level. Since the (proton) $T_{1}$ relaxation mechanism is mainly sensitive to local reorientational processes of an individual molecular group it is not surprising that the $T_{1}$ values of major metabolites reported in Table 1 are relatively close to each other without significant regional differences. The most reliable and accurate $T_{1}$ values of the singlet methyl resonances from NAA, $\mathrm{Cr} / \mathrm{PCr}$, and Cho were found to be within $1100-1750 \mathrm{~ms}$. Thus, repetition times of the order of $1500 \mathrm{~ms}$ turn out to be well suited to optimizing the signalto-noise per unit time in proton spectroscopy studies in vivo.

In general, the high local mobility of a molecule or molecular group expressed by $T_{1}$ relaxation times of the order of 1000-2000 ms may be modulated by "solvent" properties. However, in this study $T_{1}$ values seem not to be correlated to the cytosolic viscosity affected by the mean protein concentrations of cellular water. In principle, a decrease in the protein concentration from gray and white matter to thalamus and cerebellum could facilitate molecular mobility and, therefore, prolong $T_{1}$ relaxation times.

\section{$T_{2}$ Relaxation Times}

It may be expected that a different molecular or cellular organization or utilization of a metabolite in various parts of the brain would preferentially result in different $T_{2}$ relaxation times sensing "slow" overall motions of whole molecules, bound molecules, or molecular aggregates. In fact, although differences in $T_{2}$ relaxation times of cerebral metabolites were slightly more pronounced than differences in the $T_{1}$ values, no general trend may be deduced from the data in Table 2. The finding that the $T_{2}$ relaxation times of NAA in white matter regions, $(450 \pm 50) \mathrm{ms}$ in the occipital area and $(500 \pm 50) \mathrm{ms}$ in the occipital lobe, are much higher than the $(320 \pm 30) \mathrm{ms}$ found in other parts of the brain is in agreement with a recent report by others (8). It indicates particularly high mobility of NAA in white matter and may be related to its neuronal function. No significant regional variations were found for choline resonances with an average value of $(360 \pm 40) \mathrm{ms}$ and for creatine and phosphocreatine methyl resonances with a $T_{2}$ of $(220 \pm 20) \mathrm{ms}$. It is worth noting that the creatine relaxation behavior turned out to be perfectly monoexponential in contrast to what one may expect from a two-component resonance signal. Much smaller $T_{2}$ values of about $(130 \pm 30) \mathrm{ms}$ were found for the main inositol resonance probably originating from two to four of the six methine protons strongly coupled at $1.5 \mathrm{~T}$.

Another way of discussing mobility is to take the $T_{1} / T_{2}$ ratio as a measure of the degree of freedom for molecular reorientation. A ratio close to unity, i.e., $T_{2} \approx T_{1}$, is observed only for lactate, indicating free isotropic tumbling of the small molecule in the cytosol with correspondingly short correlation times. Only slight restrictions are observed for NAA and choline-containing compounds with $T_{1} / T_{2}$ ratios of about $3-3.5$. These values still refer to a high mobility of the $N$-acetyl groups of NAA and the trimethylammonium groups of free amounts of choline, phosphorylcholine, and glycerophosphorylcholine as well as of head groups of respective membrane constituents. On the other hand, the somewhat surprising finding of a $T_{1} / T_{2}$ ratio of about 
7.5 for the $N$-methyl resonance of $\mathrm{Cr} / \mathrm{PCr}$ corresponds to a certain degree of immobilization. This may be due to a more bound state, e.g., within a molecular assembly, or to a localization in a cellular compartment of viscosity higher than that in the cytosol itself. Even higher $T_{1} / T_{2}$ ratios of about 8-14 are obtained for the inositol resonance. Since the NMR visible inositols are believed to be mainly due to phosphatidyl inositols, their more restricted mobility than that of choline residues is in qualitative agreement with their difference in size and hydration.

\section{Concentrations of Cerebral Metabolites}

Table 3 summarizes relative in vivo concentrations of major cerebral metabolites in various parts of the brain as derived from localized proton NMR spectra. The numbers are normalized to 10 for total creatine in white matter. They were calculated by taking into account $T_{1}$ and $T_{2}$ relaxation times (Tables 1 and 2), spectral peak areas at 50- and 270-ms echo times, the number of protons per molecular group, the size of the VOI, and the comparability of the coil sensitivity in applications to different volunteers. In fact, all spectra in this paper have been plotted in exactly the same way and are directly comparable except for photographic reproduction.

In agreement with in vitro studies on horse and human brain using analytical techniques $(9,10)$, the relative concentration of NAA was found to be higher in gray and white matter than in the thalamus and the cerebellum. In fact, predominantly neuronal localization has been suggested for NAA, although its full role in cell metabolism remains still unknown $(11,12)$. The concentration differences between gray and white matter are not as large as previously reported. This is mainly ascribed to significant partial volume effects in the selected positions in the occipital and insular area, respectively. Again in agreement with previous fluorometric studies of rat brain (13), the regional distribution of total creatine was found to be constant. Accordingly, the ratio of NAA $/(\mathrm{Cr}+\mathrm{PCr})$ for gray and white matter of about 1.65 is markedly higher than the value of 1.25 observed in the thalamus and cerebellum.

Assuming a cerebral concentration of total creatine of about $10 \mathrm{mM}(13-15)$, the

TABLE 3

Concentrations of Major Metabolites as Detected in Localized Proton NMR Spectra of the Normal Human Brain in Vivo

\begin{tabular}{lccccc}
\hline Compound & Protons & $\begin{array}{c}\text { Insular area } \\
\text { (gray matter) }\end{array}$ & $\begin{array}{c}\text { Occipital area } \\
\text { (white matter) }\end{array}$ & Thalamus & Cerebellum \\
\hline NAA & 3 & 18 & 17 & 13 & 13 \\
Cr + PCr & 3 & 11 & $\underline{10}$ & 11 & 10 \\
Cho & 9 & 1.9 & 6.2 & 2.7 & 2.3 \\
Ins & 2 & 8.8 & 6.0 & 7.4 & 7.4 \\
\hline
\end{tabular}

Note. Relative values are normalized to the concentration of total creatine in white matter. Assuming a cerebral concentration of total creatine of about $10 \mathrm{mM}(13-15)$, the values may be read as absolute concentrations (in $\mathrm{m} M$ ). 
values given in Table 3 may be read as absolute concentrations. Thus, the most striking observation is a $17-18 \mathrm{~m} M$ NAA concentration in gray and white matter. These values are by a factor of 2-3 higher than the approximately $6 \mathrm{~m} M$ reported from a number of in vitro studies using biopsy material from different mammalian brains $(10,15-19)$. Since the NMR spectrum does not distinguish between $N$-acetyl moieties from NAA and other $N$-acetylated amino acids such as $N$-acetylglutamate and $N$ acetylaspartate glutamate, these may contribute to about $0.5 \mathrm{~m} M(20,21)$. Even higher contributions of about $2 \mathrm{mM}(22)$ may be due to $N$-acetyl moieties present in neuraminic acids found in the hydrophilic residues of glycoproteins, sialoproteins, and gangliosides. However, this is not believed to explain the considerable discrepancy of about $10 \mathrm{~m} M$ between the in vitro NAA determinations and the in vivo $N$ acetyl concentration. Since signal overlap with an eventually high glutamate resonance is also not very probable (see below), these findings need further experimental clarification. A promising approach may be studies of the time course of the NAA depletion recently observed in the early postischemic phase of stroke patients (7). Localized spectroscopy studies on animals may help to more directly investigate metabolic alterations postmortem.

A discussion of the concentration values obtained for inositol is complicated by the unknown number of protons contributing to its signal. In addition, the evaluation of $T_{2}$ relaxation times may be compromised by $J$ modulation, and the signal may contain contributions from the glycine $\mathrm{CH}_{2}$ group which occurs at the same frequency as the single inositol peak originating from multiple collapsed resonances at 1.5 T. In fact, a concentration of about $8 \mathrm{~m} M$ for phosphatidyl inositols (assuming two contributing protons) corresponds to analytical studies of human brain (23). The value is by a factor of 1.5 higher than that found in mouse brain (23) and by a factor of 1.7 lower than that reported for rabbit brain (15). The assumption of only two contributing inositol protons would double the concentration values given in Table 3. Cytosolic concentrations of inositol phosphates have recently been reported to be less than $0.1 \mathrm{~m} M$ in rat brain (24), and therefore are unable to explain the NMR observation.

Further metabolite concentrations were estimated from their signal strength at certain echo times. $T_{1}$ and $T_{2}$ relaxation times were either estimated from the in vivo results and/or measured in model solutions. Concentrations not included in Table 3 refer to taurine and aspartate and are in agreement with biochemical expectations. While aspartate turned out to be about $1.5-2.5 \mathrm{mM}$, the concentration of 1.5-2.0 $\mathrm{m} M$ for taurine in the cerebellum was higher than that in the other parts of the brain. The surprisingly low signal intensities observed for glutamate and glutamine remain an open question even in view of the known complications from extensive line splitting, multiplet overlap, and $J$ modulation artifacts. In biopsied human cerebral cortex the concentrations of both amino acids were of the order of $6 \mathrm{~m} M(9)$. Similar considerations apply to the $\beta-\mathrm{CH}_{2}$ group of aspartate in NAA.

Finally, in a preceding paper (4) we have reported a concentration of $0.4 \mathrm{mM}$ for cerebral lactate using the assumption of a $6.0 \mathrm{~m} M$ concentration for NAA. This value has also been reported in a recent human in vivo NMR study by others (8). In a number of careful attempts to more accurately determine the cerebral lactate level in 
vivo we observed a large variability of the signal strength between below detectability and a maximum value of about $1.0 \mathrm{~m} M$ in the occipital area or lobe. Although the lactate level (or other metabolite concentrations) may be affected by the physiological or functional status of the volunteers (e.g., eyes open or closed) no reproducible correlation could be established from the present studies due to actual signal-to-noise limitations.

\section{CONCLUSIONS}

Localized proton NMR spectroscopy has been demonstrated to resolve regional distribution patterns of metabolites in the normal human brain in vivo. Its high sensitivity and relatively short measuring times allow multiple spectroscopic recordings at different echo times and repetition times. NMR relaxation times $T_{1}$ and $T_{2}$ may indicate dynamical and structural differences in the cellular organization of certain metabolites in different parts of the brain. They also serve to noninvasively determine relative (and absolute) steady-state concentrations.

On the basis of reliable spatial localization, acceptable investigational times of 1$1.5 \mathrm{~h}$, and access to a large number of compounds, the present experiments show the way to a wide range of hitherto impossible biochemical and physiological studies in vivo. For example, metabolic consequences of development and aging as well as biochemical effects of anesthetics, drugs, or alcohol (25) may now be monitored directly in man. With the expected improvements in signal-to-noise and spectral resolution even functional studies of the cerebral metabolism are within future possibilities. Medical applications of localized proton NMR spectroscopy will considerably improve the diagnostic specificity originally expected from MRI. There is hope to optimize therapeutic efficacy by directly monitoring biochemical response. Localized proton spectroscopy as a diagnostic tool for a noninvasive metabolic characterization of cerebral infarcts and tumors bears a great potential for replacing needle biopsy.

\section{ACKNOWLEDGMENT}

Financial support by the Bundesminister für Forschung und Technologie (BMFT) of the Federal Republic of Germany (Grant 01 VF 8606/6) is gratefully acknowledged.

\section{REFERENCES}

1. J. W. Prichard and R. G. Shulman, Annu. Rev. Neurosci. 9,61 (1986).

2. J. Frahm, K. D. MER boldt, AND W. HÄNICKE, J. Magn. Reson. 72, 502 (1987).

3. M. L. Gyngell, J. Frahm, K. D. Merboldt, W. Hänicke, and H. Bruhn, J. Magn. Reson. 77, 596 (1988).

4. J. Frahm, M. L. Gyngell, H. Bruhn, K. D. Merboldt, W. Hänicke, and R. Sauter, Magn. Reson. Med. 9,79 (1989).

5. J. Frahm, H. Bruhn, M. L. Gyngell, K. D. Merboldt, W. Hänicke, and R. Sauter, Japan. $J$. Magn. Reson. Med. 8, 49 (1988).

6. H. Bruhn, J. Frahm, M. L. Gyngell, K. D. Merboldt, W, Hänicke, R. Sauter, and C. HamBURGER, Radiology, in press.

7. H. Bruhn, J. Frahm, M. L. Gyngell, K. D. Merboldt, W. Hänicke, and R. Sauter, Magn. Reson. Med. 9, 126 (1989).

8. C. C. Hanstock, D. L. Rothman, J. W. Prichard, T. Jue, and R. G. Shulman, Proc. Natl. Acad. Sci. USA 85, 1821 (1988). 
9. T. L. Perry, S. Hansen, K. Berry, C. MoK, AND D. Lesk, J. Neurochem. 18, 521 (1971).

10. H. H. Tallan, J. Biol. Chem. 224, 41 (1957).

II. K. B. JACOBSON, J. Gen. Physiol. 43, 323 (1959).

12. J. V. NADLER AND J. R. COOPER, J. Neurochem. 19, 2091 (1972).

13. L. D. Lewis, B. LJunggren, R. A. Ratcheson, AND B. K. Siesjö, J. Neurochem. 23, 673 (1974).

14. R. L. Veech, J. W. R. Lawson, N. W. Cornell, AND H. A. KrebS, J. Biol. Chem. 254, 6538 (1979).

15. O. A. C Petroff, T. OGino, AND J. R. Alger, J. Neurochem. 51, 163 (1988).

J6. J. K. TeWs, S. H. Carter, P. D. RoA, AND W. E. Stone, J. Neurochem. 10, 641 (1963).

17. R. K. Shaw AND J. D. HeINE, J. Neurochem. 12, 151 (1965).

18. M. C. Fleming AND O. H. Lowry, J, Neurochem. 13, 779 (1966).

19. J. V. NADLER AND J. R. COOPER, J. Neurochem. 19, 313 (1972).

20. A. Curatolo, P. D'Arcangelo, A. Lino, and A. Brancati, J. Neurochem. 12, 339 (1965).

21. K. L. REICHELT AND F. FonNUM, J. Neurochem. 16, 1409 (1969).

22. H. MCIlwain and H. S. BaChelaRD, "Biochemistry and the Central Nervous System," 5th ed., p. 314, Churchill Livingston, Edinburgh, 1985.

23. K. NarūMi, M. ARita, M. Kitagawa, A. KūMazawa, and T. Tsūmita, Japan. J. Exp. Med. 39, 399 (1969).

24. J. L. MeEK, Proc. Natl. Acad. Sci. USA 83, 4162 (1986).

25. C. C. Hanstock, D. L. Rothman, R. G. Shulman, E. Novotny, O. A. C Petroff, and J. W. PriCHARD, "7th Annual Meeting, Society of Magnetic Resonance in Medicine, August 20-26, 1988, San Francisco," p. 1071. [Book of Abstracts]. 